\title{
A Weighted Multilevel Median Filtering Algorithm
}

\author{
Dehai Shen $^{\mathrm{a}}$, Jian Hou ${ }^{\mathrm{b}}, \mathrm{Xu} \mathrm{E}^{\mathrm{c}}$, Longchang Zhang ${ }^{\mathrm{d}}$
}

College of Information Science and Technology, Bohai University, Jinzhou, Liaoning 121013, China

asdh25@sohu.com, bjian_hou@163.com, exu21@163.com, d22427291@qq.com

Keywords: mixed noise, multilevel median filter, Weight.

Abstract. In order to remove the mixed noise in an image, proposed a weighted filtering algorithm. The algorithm divided a $3 \times 3$ filtering window into six horizontal and vertical sub-windows, found out every median pixel of pixels in every sub-window and the median of these median pixels, calculated the absolution of difference between these median pixels and their median, then used these absolutions and their mean to calculate corresponding weight of every median pixel in sub-window according to normalizing method, used median pixel in every sub-window to multiple by its weight and sum, the result was taken as the center pixel filtering out. Experiments show that the proposed algorithm has better filtering performance for images mixed with impulse noise and Gauss noise than some others algorithms.

\section{Introduction}

During the imaging, quantizing, transmitting, digital images are often contaminated by noise, and become blurred, which seriously affects the subsequent processing, such as feature extraction, image segmentation and image recognition and so on. Image denoising aims to eliminate all kinds of noise interference, at the same time, keeps the detail information of the image, and so provides the good accuracy for subsequent processing. Impulse noise and Gauss noise are the most common, for the two kinds of noise, domestic and foreign scholars proposed many filtering algorithms, such as traditional mean filtering algorithm, traditional median filtering algorithm, multilevel median filtering algorithm and some improved filtering algorithms based on them [1-4].

Traditional mean filtering algorithm computes the average value of all pixels in filtering window, replace the central pixel value in window [5], has good denoising effect for Gauss noise, but can't remove the impulse noise well. Traditional median filtering algorithm uses the median of all pixels in the filtering window to replace the central pixel value in the window, has good denoising effect for impulse noise, and can preserve the image details well, but with the increasing of noise density (greater than 50\%), its denoising performance will decline sharply. Multilevel median filtering algorithms in $[3,4]$ divide filtering window into many sub-windows, use the median of all medians in all sub-windows to replace the gray of central pixel in filtering window, have better denoising performance and detail preserving ability than traditional median filtering algorithm for impulse image, but for Gauss noise, effect is not idea. In this paper, we propose a weighted multilevel median filtering algorithm .The algorithm divides a $3 \times 3$ filtering window into six horizontal and vertical sub-windows, then for every median of sub-windows, use weighted method to compute the filtering output of the central pixel in filtering window, the algorithm has good denoising effect for image mixed with impulse noise and Gauss noise.

\section{Multilevel median filtering algorithm}

As an extension of median filtering algorithm, the multilevel median filtering is simple and 
efficient, and has excellent detail preserving performance, such as all kinds of fine line. Supposes $x(m, n)$ indicate the gray of pixel at $(m, n)$ in a noise image, its principle is described as follows.

Divides the filtering window, in a square filtering window, lets $L$ is its length $(L=2 N+1), N$ is a integer greater than zero. Suppose $x(m-i, n-j)$ as the central pixel, $-N \leq i, j \leq N$, divides the filtering window into four sub-windows: $W_{1} 、 W_{2} 、 W_{3}$ and $W_{4}$, indicate one-dimensional sub-window of $0^{\circ}$ direction、 $90^{\circ}$ direction、 $45^{\circ}$ direction and $135^{\circ}$ direction respectively. Let $M_{1}(m, n) 、 M_{2}(m, n)$ 、 $M_{3}(m, n)$ and $M_{4}(m, n)$ indicate the median of four sub-windows respectively, the formula is shown in Eq.1.

$$
M_{k}(m, n)=\operatorname{median}\left[x(i, j) \in W_{k}(m, n)\right], k=1,2,3 、 4
$$

Let $M_{\max }(m, n) 、 M_{\min }(m, n)$ indicates maximum and minimum of four medians $M_{1}(m, n) 、 M_{2}(m$, $n) 、 M_{3}(m, n)$ and $M_{4}(m, n)$ respectively, the formulas are shown in Eq.2 and Eq.3.

$$
\begin{aligned}
& M_{\text {max }}(m, n)=\max \left[M_{1}(m, n), M_{2}(m, n), M_{3}(m, n), M_{4}(m, n)\right] \\
& M_{\text {min }}(m, n)=\min \left[M_{1}(m, n), M_{2}(m, n), M_{3}(m, n), M_{4}(m, n)\right]
\end{aligned}
$$

The output of algorithm can be expressed in Eq.4, the gray value of central pixel in filtering window is replaced by $f^{\prime}(m, n)$.

$$
f^{\prime}(m, n)=\operatorname{median}\left[M_{\text {max }}(m, n), M_{\text {min }}(m, n), x(m, n)\right]
$$

The multilevel median filtering algorithm has good performance of eliminating impulse noise, and preserves the image details well, but it can't eliminate the Gauss noise.

\section{Improved multilevel median filtering algorithm}

To remove the mixed noise, proposed a weighted multilevel median filtering algorithm,we use the method in [6] to divide the filtering window, the sub-windows are shown in Fig.1.

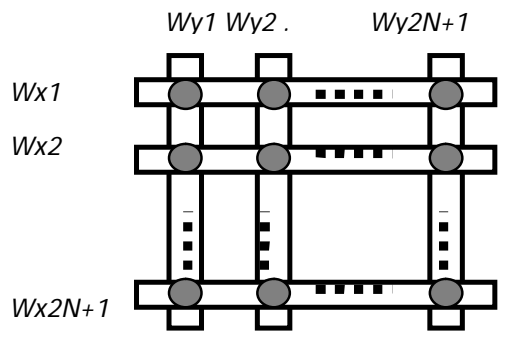

Fig 1 filtering sub-windows in this paper

In Fig. 1, the filtering window is divided into $2 L$ vertical direction and horizontal sub-windows, $L$ is the length of square filtering window. The steps of filtering process as follows:

1) Obtaining the median pixels and their median. In this paper, $f(i, j)$ indicates noise image, we suppose the filtering window size is $3 \times 3,(i, j)$ is center of the window, there are six sub-windows, 
finds the median $M_{k}$ of every sub-window, $k=1 、 2 、 3 、 4 、 5 、 6$, then computes the median $M(i, j)$ of these medians as shown in Eq.5.

$$
M(i, j)=\operatorname{median}\left[M_{1}(i, j), M_{2}(i, j), M_{3}(i, j), M_{4}(i, j), M_{5}(i, j), M_{6}(i, j)\right]
$$

2) Computing the weights. First, calculates the absolutions of deference pixels $\left(M_{1}-M_{6}\right)$ and their median $(M)$, and then gets the average of these absolutions, uses the result as optimization threshold T, the formula is shown in Eq.6. Second, uses the method in Eq.7 to computes the weights of pixels $\left(M_{1}-M_{6}\right)$.

$$
\begin{aligned}
& \mathrm{T}=\frac{\sum_{k=1}^{6}\left|M_{k}-M\right|}{6} \\
& w_{k}(i, j)=\frac{1 /\left(1+\operatorname{Max}\left(\left|M_{k}-M\right|, \mathrm{T}\right)\right)}{\sum_{k=1}^{6} 1 /\left(1+\operatorname{Max}\left(\left|M_{k}-M\right|, \mathrm{T}\right)\right.}
\end{aligned}
$$

In Eq.7, $w_{k}(i, j)$ is the weight of median pixels $\left(M_{1}-M_{6}\right)$, in this paper, uses threshold method to calculate the weights of median pixels in sub-window. The weight of every median pixel is determined by maximum between absolution of $\left(M_{1}-M\right)$ and threshold T. If don't use threshold T, then the deference of noise pixel whose gray close to $M$ will be very small, the corresponding weight will be very great, so will expand the influence of noise.

3) Weighting and filtering out. The median pixels $\left(M_{1}-M_{6}\right)$ multiple by their corresponding weights and sum up, the result is as the filter output of central pixel in filtering window, the method is shown in Eq.8.

$$
f(i, j)=\sum_{k=1}^{6} M_{k} \cdot w_{k}(i, j)
$$

\section{Verification experiment and the effect analysis}

Selects standard gray image lena with $256 \times 256$ pixels as initial image to make experiments, we mix different density impulse noise (salt \& pepper noise) and Gauss noise in initial image each time, use traditional median filtering algorithm (MedF), traditional mean filtering algorithm (MeaF), multilevel median filtering algorithm(MLM+) and proposed algorithm(NEWF) to filter noise image respectively, the size of filtering window of every algorithm is $3 \times 3$. The results are shown in Fig. 2 and Fig.3, the PSNR of these algorithms are shown in table1.

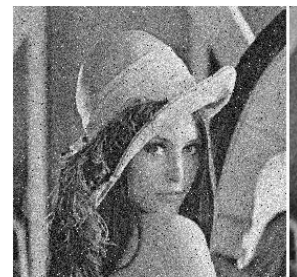

(a)

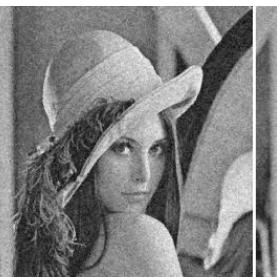

(b)

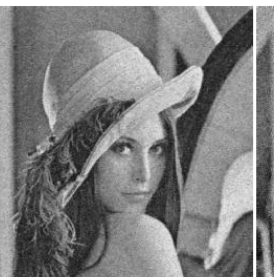

(c)

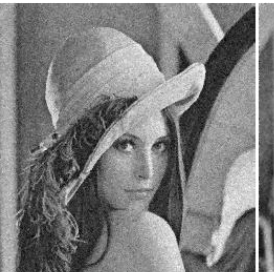

(d)

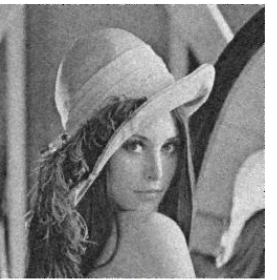

(e)

Fig 2 filtering image with low density Gauss noise and salt \& pepper noise 


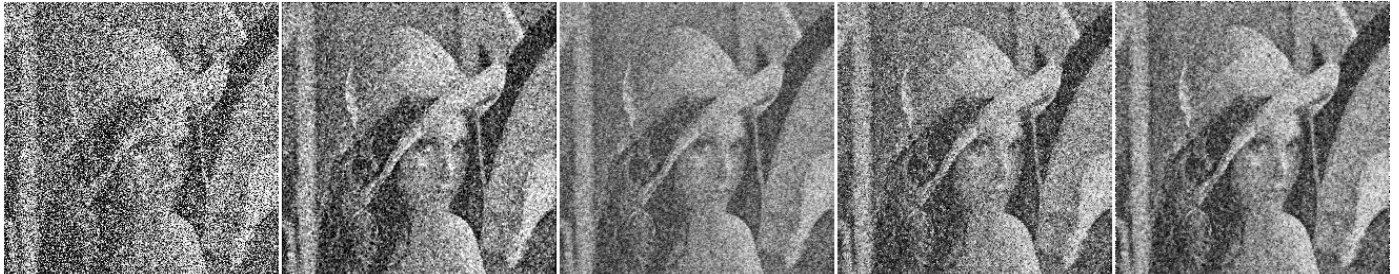

(a)

(b)

(c) (e)

Fig 3 filtering image with high density Gauss noise and salt $\&$ pepper noise

In Fig.2-Fig.3, (a) is mixed noise image; (b) is MedF filtering image; (c) is MeaF filtering image; (d) is MLM+ filtering image;(e) is NEWF algorithm image.

In Fig.2, the initial image is mixed with Gauss noise of variance is 0.01 (mean is 0 ) and salt and pepper noise of 1\%. In Fig.3, the initial image is mixed with Gauss noise of variance is 0.01 (mean is 0 ) and salt and pepper noise of $30 \%$. We can see when the density of mixed noise is low, four algorithms have good denoising performance, but with increasing of the mixed noise, the denoising performance of traditional mean filtering algorithm and multilevel median filtering algorithm decrease rapidly, but the proposed algorithm have stable denoising ability, the filtering image is clear than other algorithms, and has good details preserving performance.

Table 1 the PSNR comparisons of four algorithms

\begin{tabular}{llllllll}
\hline Gauss noise & 0.01 & 0.02 & 0.03 & 0.04 & 0.05 & 0.06 & 0.07 \\
Salt \& pepper noise & $1 \%$ & $10 \%$ & $20 \%$ & $30 \%$ & $40 \%$ & $50 \%$ & $60 \%$ \\
\hline MeaF & 27.23 & 22.58 & 20.29 & 18.41 & 17.07 & 16.00 & 15.13 \\
MedF & 26.59 & 23.07 & 20.80 & 18.03 & 15.97 & 14.35 & 12.90 \\
MLM+ & 25.72 & 22.14 & 19.50 & 17.53 & 15.94 & 14.65 & 13.62 \\
NEWF & 28.05 & 24.55 & 21.86 & 19.84 & 18.07 & 16.66 & 15.39 \\
\hline
\end{tabular}

From these PSNR data in table 1, we can see that the PSNR of MedF and MLM+ is lower than proposed algorithm and MeaF algorithm in deferent density mixed noise image, and the proposed algorithm has highest PSNR, Table 1 proves that the algorithm in this paper has stronger denoising performance and detail preserving ability objectively.

\section{Conclusions}

In order to remove the mixed noise in an image, proposed a weighted multilevel median filtering algorithm. The algorithm divides the filtering window into some sub-windows, and uses threshold method to calculate the weights of median pixels in sub-windows. Experiments show that the improved algorithm has stronger denoising performance and details preserving ability than other algorithms.

\section{Acknowledgement}

The project is sponsored by National Nature Science Foundation under Grant 61473045.

\section{References}

[1] ZHANG Heng, LEI Zhi-hui, DING Xiao-hua. An Improved Method of Median Filter. Journal of Image and Graphics, vol.9, no.4, p. 408-411, 2004, (In Chinese). 
[2] Zhu Shihu, You Chunxia , A MODIFIED AVERAGE FILTERING ALGORITHM. Computer Applications and Software, vol.30, no.12, p. 97-99,116,2013, (In Chinese).

[3] Nieminen A, Heinonen P, Neuvo Y. A new class of detail preserving filters for image processing. IEEE Tran on PAMI, vol.9, no.1, p. 74-79,1978.

[4] Arce G R, Foster R E. Detail preserving ranked order based filters for image processing. IEEE Tran on ASSP, vol.37, no.1, p. 83-98, 1989.

[5] Mei-Sen Pan, Jing-Tian Tang, Xiao-Li Yang. An Adaptive Median Filter Algorithm Based on B-spline Function. International Journal of Automation and Computing, vol.8, no.1, p. 92-99, 2011, (In Chinese).

[6] Shen De-hai, LIU Da-cheng, XING Tao. A Multilevel Median Filter Algorithm Based On Vertical And Horizontal Windows Related. Computer Science, vol.39, no.5, p. 246-248, 2012, (In Chinese). 